\title{
BMJ Open Examining the effects of creatine supplementation in augmenting adaptations to resistance training in patients with prostate cancer undergoing androgen deprivation therapy: a randomised, double-blind, placebo-controlled trial
}

\author{
Ciaran M Fairman, ${ }^{\oplus 1,2}$ Krissy L Kendall, ${ }^{2}$ Robert U Newton, ${ }^{1,2,3}$ Nicolas H Hart, ${ }^{\oplus 1,2,4}$
} Dennis R Taaffe, ${ }^{1,2,3}$ Raphael Chee, ${ }^{2,5}$ Colin I Tang, ${ }^{6}$ Daniel A Galvão ${ }^{\oplus 1,2,3}$

To cite: Fairman CM, Kendall KL, Newton RU, et al. Examining the effects of creatine supplementation in augmenting adaptations to resistance training in patients with prostate cancer undergoing androgen deprivation therapy: a randomised, double-blind, placebocontrolled trial. BMJ Open 2019;9:e030080. doi:10.1136/ bmjopen-2019-030080

- Prepublication history for this paper is available online. To view these files, please visit the journal online (http://dx.doi org/10.1136/bmjopen-2019030080)

Received 26 February 2019 Revised 13 August 2019 Accepted 06 September 2019

Check for updates

(C) Author(s) (or their employer(s)) 2019. Re-use permitted under CC BY-NC. No commercial re-use. See rights and permissions. Published by BMJ.

For numbered affiliations see end of article.

Correspondence to

Dr Ciaran M Fairman;

c.fairman@ecu.edu.au

\section{ABSTRACT}

Introduction Creatine supplementation has consistently been demonstrated to augment adaptations in body composition, muscle strength and physical function in a variety of apparently healthy older adults and clinical populations. The effects of creatine supplementation and resistance training in individuals with cancer have yet to be investigated. This study aims to examine the effects of creatine supplementation in conjunction with resistance training on body composition, muscle strength and physical function in prostate cancer patients undergoing androgen deprivation therapy.

Methods and analysis This is a randomised, doubleblind, placebo-controlled trial designed to examine the effects of creatine supplementation in addition to resistance training in patients with prostate cancer receiving androgen deprivation therapy. Both supplement and placebo groups will receive a 12-week supervised exercise programme comprising resistance training undertaken three times per week. The primary endpoint (fat-free mass) and secondary endpoints (fat mass, per cent body fat, physical fitness, quality of life and blood biomarkers) will be assessed at baseline and immediately following the intervention.

Ethics and dissemination The Human Research Ethics Committee of Edith Cowan University approved this study (ID: 22243 FAIRMAN). If the results of this trial demonstrate that creatine supplementation can augment beneficial adaptations of body composition, physical function and/or psychosocial outcomes to resistance training, this study will provide effect sizes that will inform the design of subsequent definitive randomised controlled trials. The results of this study will be published in peer-reviewed journals and presented at various national and international conferences. Trial registration number ACTRN12619000099123
Strengths and limitations of this study

This is the first trial designed to examine the effects of creatine supplementation and resistance training on body composition in patients with prostate cancer, addressing a critical area of concern in this population.

- Both groups receive an exercise intervention which may enhance recruitment affording all patients the benefits of exercise and negates questions surrounding attention control.

- The study is limited to men with prostate cancer on androgen deprivation therapy and results may not be generalised to other populations.

- The inclusion of dietary analysis at baseline, 6-week midpoint and 12-week follow-up will offer additional insight into dietary habits of participants that may influence muscle hypertrophy (ie, protein intake).

- The lack of follow-up assessments limits information on the sustainability of benefit.

\section{INTRODUCTION}

Androgen deprivation therapy (ADT) is frequently incorporated into the management of men with localised and advanced prostate cancer (PCa). ${ }^{1}$ Unfortunately, the catabolic effects of ADT result in loss of fat-free mass (FFM), increased fat mass, reduced muscle strength and lower bone mineral density (BMD). ${ }^{2-5}$ In turn, these adverse effects of ADT are linked with functional decline (ie, reduced walking speed, ability to rise from a chair or climb stairs, etc), frailty, and increased risk for cardiovascular disease, metabolic syndrome and osteoporosis. ${ }^{6}$ Thus, patients with PCa endure long and lingering 
impacts on physical function, health status and quality of life (QOL) that accompany ADT as a 'trade-off' for more effective cancer control and extended longevity.

Resistance training has gained increasing attention in the recent years, with the results from numerous studies supporting resistance training as a promising strategy to counteract declining body composition, BMD and physical function mediated by ADT. ${ }^{7-11}$ Nevertheless, there remains a pronounced exacerbation of normal ageing (decline in lean body mass, muscular strength and endurance) as a result of ADT, resulting in an accelerated trajectory towards a 'disability' condition (ie, the difficulty or reduced ability to perform activities essential to independent living). ${ }^{12}$ Consequently, there is an urgent need for strategies to augment resistance training adaptations and further target the worsening of body composition, muscle strength and physical function in this population. ${ }^{613-15}$

More recently, there has been greater emphasis on the need for multimodal strategies combining exercise and dietary/supplementation interventions to more aggressively target.

FFM in patients with cancer. ${ }^{16}$ Creatine $(\mathrm{Cr})$ is one of the most widely studied supplements (SUPPs), with research consistently demonstrating greater improvements in FFM, strength and physical function when combined with resistance training, compared with that achieved with resistance training alone. ${ }^{17-22}$ Further, emerging research has demonstrated the efficacy of $\mathrm{Cr}$ supplementation for improving FFM, BMD, muscle strength and physical function in older adults or clinical populations with similar muscle wasting characteristics to cancer, such as muscular dystrophy and HIV. ${ }^{23-30}$

Cr supplementation increases intramuscular stores of phosphocreatine (PCr), a high-energy phosphate that plays a critical role in energy provision during exercise. ${ }^{31}$ It is hypothesised that $\mathrm{Cr}$ uptake by skeletal muscle is modulated by muscle activity. ${ }^{32}$ Importantly, the ability to sustain exercise effort is dependent on PCr availability, which diminishes with intense exercise. Increases in $\mathrm{PCr}$ stores may contribute to an accelerated resynthesis of adenosine triphosphate (ATP) during exercise. ${ }^{19} 20$ Consequently, the ergogenic effects of Cr supplementation are likely a result from an increase in overall training volume and/or quality, ${ }^{19}{ }^{20}$ due to increases in intramuscular PCr stores $^{32} 33$ enhancing exercise capacity. Taken collectively, there is the potential that Cr supplementation may enhance the quality and/or quantity of resistance training in patients with PCa, leading to augmented adaptations in FFM, muscle strength and/or function. ${ }^{16}$ Given the overwhelming evidence of $\mathrm{Cr}$ supplementation in other populations, there is large therapeutic potential for its use in patients with PCa. ${ }^{16}$

The primary aim of this study is to examine the effects of $\mathrm{Cr}$ supplementation in conjunction with resistance training on body composition, muscle strength and physical function in patients with PCa undergoing ADT. It is hypothesised that resistance training plus $\mathrm{Cr}$ will lead to greater improvements in FFM, muscle strength, QOL and physical function than that of resistance training alone. The results of this study will provide insights into the effects of Cr supplementation as a potential low-cost therapeutic agent to target FFM in men with PCa receiving ADT.

\section{METHODS}

\section{Study design}

This is a randomised, double-blind, placebo (PLA)-controlled trial designed to examine the effects of $\mathrm{Cr}$ supplementation in addition to resistance training in patients with PCa receiving ADT. Both SUPP and PLA groups will receive a 12-week supervised exercise programme comprising of resistance training undertaken three times per week. In addition, participants in the SUPP group will receive a daily dose of Cr: $20 \mathrm{~g}$ / day during a loading phase of 5 days (the last 5 days of the familiarisation period, described in detail later) and then $5 \mathrm{~g}$ /day thereafter for the duration of the 12-week exercise intervention. Participants in the PLA group will follow the same dosing protocol with dextrose. There will be a 2-week testing/familiarisation period (detailed further in this manuscript) prior to the beginning of the intervention, with post-testing occurring in the 2 weeks following the intervention. Consequently, the total length of the study is approximately 16 weeks. An overview of the study design and participant flow is depicted in figure 1.

\section{Participants}

Fifty-six men $(n=56)$ with localised or locally advanced $\mathrm{PCa}$, who are currently on ADT (and expected to remain on $\mathrm{ADT}$ for at least 4 months postrecruitment), will be eligible to enrol in this study. Participants will be excluded if they have any visceral or bone metastases (ie, advanced or castrate-resistant PCa); are being treated for any secondary or other cancers or are currently participating in regular structured resistance training ( $>2$ days/week for 6 months) or are receiving medications known to alter body composition (ie, corticosteroids, metformin, etc). Individuals who are participating in regular structured aerobic training ( $>2$ days a week) will not be excluded. Baseline activity will be recorded and participants enrolled in the study will be asked not to modify outside activity. Additionally, participants will be excluded if they have taken $\mathrm{Cr}$ in the previous 6 months.

\section{Recruitment}

Participants will be recruited through a variety of avenues in Perth, Western Australia. Primarily, participants will be approached by their attending specialist, who will discuss details of the trial and provide individuals with a study information sheet and referral to the study coordinator, who will verify eligibility by a telephone conversation. Additionally, flyers will be posted throughout local medical clinics in the Perth area. All participants must 
16-weeks Protocol

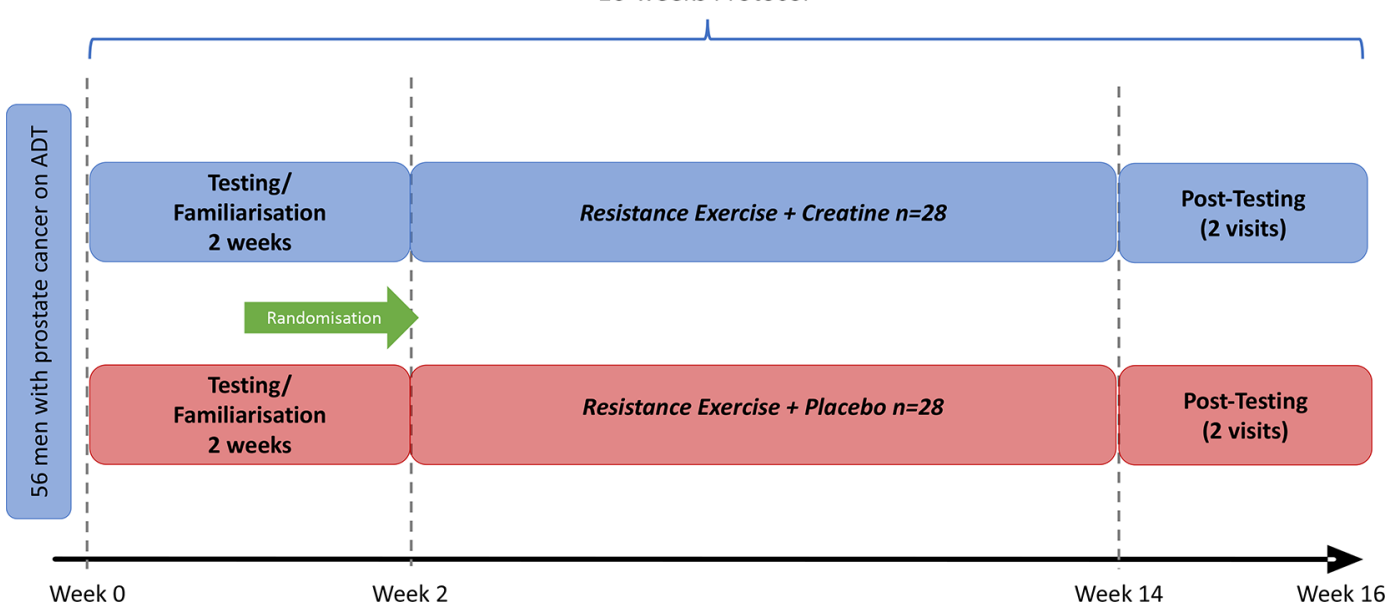

Figure 1 Overview of study design.

receive physician clearance and provide informed consent prior to participation in the trial. Proposed participant flow throughout the study is presented in figure 2 .

\section{Measurements}

Baseline and follow-up assessments will be undertaken within 2 weeks of the participant beginning or completing the intervention, respectively. An overview of assessments and timeline of study activities is outlined in table 1 .

\section{Randomisation}

Following baseline assessments (ie, on day 4 of familiarisation phase, following completion of strength and physical function testing), consenting participants will be randomised in a ratio of 1:1 to either SUPP or PLA using computer-generated random assignment by a member of the study team with no contact with participants. Participants will be stratified according to time on ADT ( $\leq 6$ and $>6$ months), age ( $\leq 65$ and $>65$ years) and randomly assigned to one of the two groups. All participants and members of the study team will be blinded to group allocation.

\section{Primary endpoint}

\section{Fat free mass}

Whole body FFM and appendicular (lower and upper limb) skeletal muscle mass will be assessed using dual-energy X-ray absorptiometry (Horizon A, Hologic, Massachusetts, USA) in accordance with Hart et al. ${ }^{34}$ Participants will be asked to avoid strenuous exercise for 24 hours prior to testing. Further, they will be instructed to avoid the consumption of food and water 4 hours and 1 hour prior to testing, respectively.

\section{Secondary endpoints}

Muscle strength

Dynamic muscle strength will be assessed using one-repetition maximum (1RM) tests for chest press, seated row and leg press exercises. ${ }^{715} 1 \mathrm{RM}$ tests are the standard by which muscular strength is evaluated and have been used in prior investigations in individuals with cancer. ${ }^{715}$
Participants will be asked to perform a general warmup, followed by two exercise-specific warm-up sets (4-6 repetitions), separated by $90-180$ s. Participants will then be asked to complete a maximal attempt (the maximal amount of weight an individual can lift with proper technique through a full range of motion). Several maximal lifts will be attempted (separated by $3-5 \mathrm{~min}$ ) until the participant reaches a weight that cannot be lifted using proper technique. Efforts will be made to ensure a 1RM is reached in as few attempts as possible.

\section{QOL and fatigue}

Health-related QOL for general health, pain, vitality, social functioning, emotional role and mental health will be assessed using the Medical Outcomes Short Form 36 V.2. ${ }^{35}$ Cancer-specific QOL indices will be measured using the European Organisation for Research and Treatment of Cancer (EORTC) Quality of Life Questionnaire-C30 and EORTC PR-25, ${ }^{36} 37$ assessing physical, emotional, cognitive and sexual functioning, along with PCa-specific symptoms. Cancer-related fatigue will be assessed using the Functional Assessment of Chronic Illness Therapy-Fatigue questionnaire. ${ }^{38}$

\section{Physical fitness}

A battery of standard tests will be used to assess physical function, including the $400 \mathrm{~m}$ walk test; the timed up-and-go test, and repeated chair rise test to assess physical function. ${ }^{715}$ The $400 \mathrm{~m}$ walk test will be completed in a corridor/hallway with two cones spaced $20 \mathrm{~m}$ apart. Individuals will be asked to walk around the cones as quickly as possible, completing a total of 10 laps, with the total time to completion recorded. The timed up-and-go test will be completed with a chair and a cone placed $3 \mathrm{~m}$ away. Individuals will be instructed to begin the test seated in the chair, then instructed to get up, walk around the cone and sit back down, with the total time for 1 lap recorded. For the timed-up-and-go test and repeated chair rise, the chair will be placed against a wall to ensure the safety of participants. The repeated chair rise will be completed 


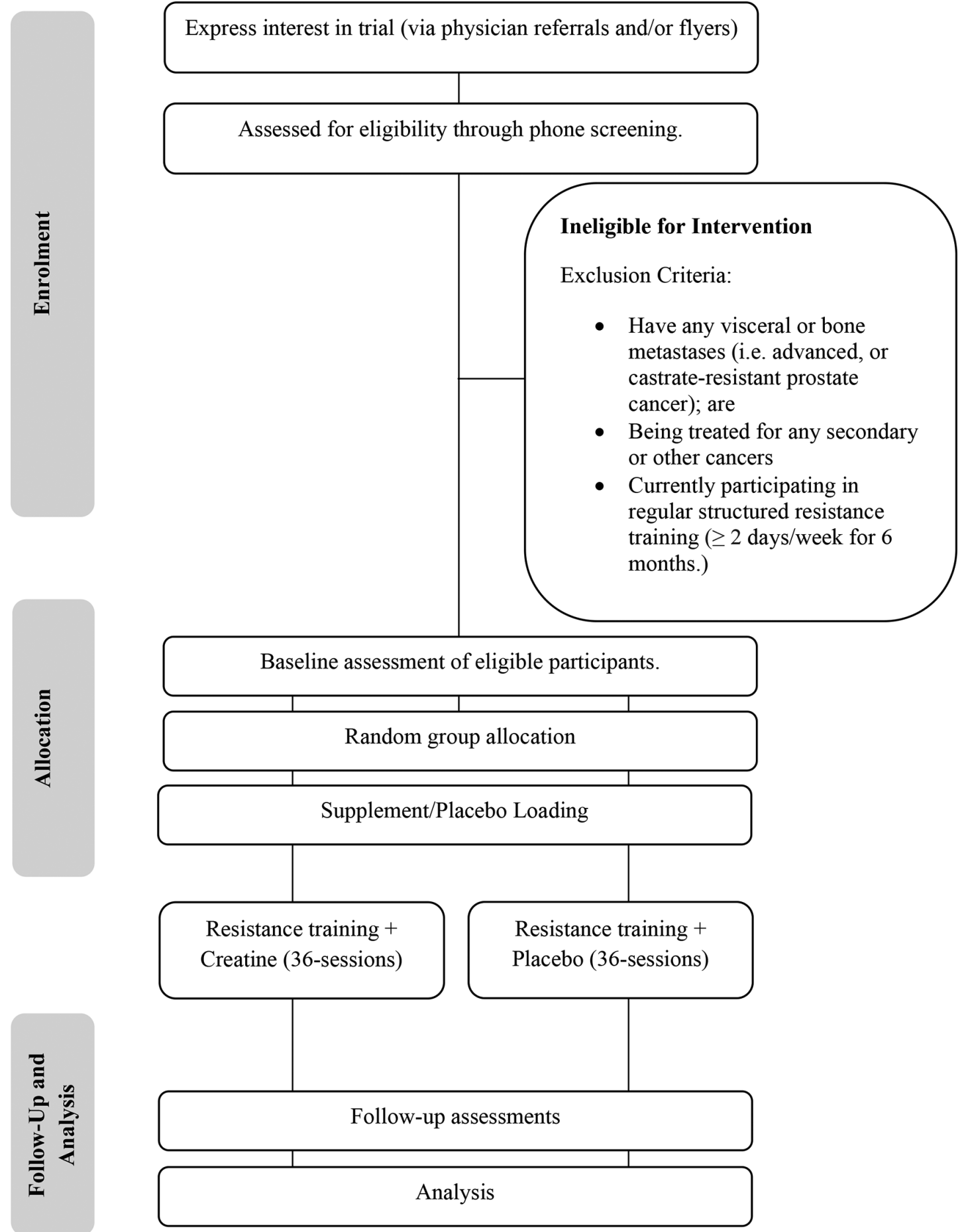

Figure 2 CONSORT diagram. CONSORT, Consolidated Standards of Reporting Trials.

by having the patient sit in a chair, and asked to rise to a full standing position, sit back down and repeat five times as quickly as possible, with the total time recorded as the performance measure.

\section{Other measures}

Medical history

Information pertaining to cancer-specific (treatment history, time on treatment, stage of cancer and time since diagnosis, etc) and general medical history (comorbidities, lifestyle, concomitant medications and other chronic diseases, etc) will be obtained via questionnaires prior to baseline testing, and will be actively monitored through out the trial to capture any changes.

\section{Diet recall}

Additionally, participants will be asked to complete a 3-day food record of the days preceding baseline testing. They will be asked to replicate this diet for post-testing. Further, a 3-day diet recall will be completed at midpoint to gain further information about dietary changes that may occur throughout the study. 
Table 1 Overview of testing and timeline of study activities

\begin{tabular}{|c|c|c|c|c|}
\hline Time point & Week 1 & Week 2 & Week 15 & Week 16 \\
\hline Informed consent & $x$ & & & \\
\hline Medical history and demographics & $x$ & & & \\
\hline Body composition (DXA) & $x$ & & $x$ & \\
\hline 1RM, (leg press, chest press, seated row) & & $x$ & & $x$ \\
\hline Quality of life (SF-36, EORTC-QLQ-C30; EORTC-PR25; FACIT-F) & $x$ & & $x$ & \\
\hline Gastrointestinal questionnaire & $x$ & $x$ & $x$ & \\
\hline Blood draw & $\mathrm{x}$ & & $x$ & \\
\hline
\end{tabular}

DXA, dual-energy X-ray absorptiometry; EORTC-PR25, European Organisation for Research and Treatment of Cancer Prostate Cancer Module; EORTC-QLQ-C30, European Organisation for Research and Treatment of Cancer Quality of Life Questionnaire; FACIT-F, Functional Assessment of Chronic Illness Therapy-Fatigue; 1RM, one repetition maximum; SF-36, Short Form 36.

\section{Anthropometrics}

Height will be recorded to the nearest $1 \mathrm{~cm}$ using a wallmounted stadiometer (Model 222, Seca, Hamburg, DE), with body mass recorded to the nearest $0.1 \mathrm{~kg}$ using an electronic scale (AE Adams CPW Plus-200, Adam Equipment, Connecticut, USA).

\section{Adverse effects}

In order to examine any potential side effects of supplementation in this population, fasted blood samples will be collected and analysed commercially by an accredited National Association of Testing Authorities laboratory (Australian Clinical Labs, Perth, Australia) for creatinine at baseline and follow-up. Additionally, participants will be asked to complete questionnaires related to gastrointestinal distress (nausea, bloating and upset stomach, etc) and muscle cramping. ${ }^{19}$ Accredited exercise physiologists (AEPs, Exercise and Sport Science Australia) will supervise the exercise sessions, ensuring correct technique and appropriate progression, minimising the risk of adverse events. Consequently, a data monitoring and safety committee has not been appointed. However, participants will be consistently monitored for any adverse effects, and other disease-related or exercise-related adverse events using adverse event logs across each patient's on-trial period, throughout the testing and training sessions. Adverse events will be documented in accordance with Exercise Medicine Research Institute (EMRI) standards and reported to the HREC. Additionally, the participant will be referred to their general practitioner or specialist, as appropriate for a medical assessment of any adverse event. Participants are free to withdraw from the study at any time.

\section{Familiarisation and baseline testing}

All participants enrolled in the study will undergo a 2-week familiarisation/testing period, where they will complete baseline testing, begin the supplementation protocol and receive instruction on safe and appropriate exercise technique. Specifically, participants will undergo baseline body composition testing on day 1 . Following 2 days of learning correct exercise technique, they will perform baseline strength and physical function testing (day 4). They will also begin the 5-day loading protocol for supplementation (outlined below) on day 4 in an effort to ensure intramuscular $\mathrm{Cr}$ stores are saturated prior to the beginning of the exercise programme. Further details on the familiarisation/testing phase are outlined in table 2.

\section{Exercise programme}

Participants assigned to each arm will attend a total of 36 supervised exercise sessions for 12 weeks (thrice weekly, with a minimum 48 hours rest between sessions). Currently, the EMRI has four exercise clinics across Perth. Participants will be given the option to choose the clinic that is closest/most convenient for them to complete their training and all sessions will be supervised by an AEP. The resistance training programme will comprise eight exercises targeting large muscle groups of the body (leg press, deadlift, step up, chest press, push-ups, shoulder press, lat pulldown and seated row). The initial loading will be equivalent to $\sim 65 \% 1 \mathrm{RM}$ (3 sets of 12 reps), progressing towards $\sim 80 \%$ 1RM (4 sets of 8 reps). Loading will be progressed throughout the programme using the ' 2 for 2 rule', whereby if a participant can complete two additional reps on the last set of an exercise for two consecutive sessions, the weight for an exercise ( $5 \%$ to $10 \%$ for upper body; $\sim 10 \%$ to $15 \%$ for lower body) will be increased. Concurrently, repetitions will gradually decrease across the programme to match the increase in weight. This type of resistance training protocol (ie, exercises that stimulate large muscle groups, multiple sets and short rest) has been recommended to enhance the hypertrophic response to training. ${ }^{39} 40$ The programme will be autoregulated where variations in participants' fatigue, recovery, energy and physical capacity will be used to adjust each training session. This model of autoregulation 
Table 2 Familiarisation and testing phase

\section{Week 1 familiarisation}

\begin{tabular}{|c|c|c|}
\hline Day 1 & Day 2 & Day 3 \\
\hline $\begin{array}{l}\text { 3-day food record } \\
\text { DXA } \\
\text { Questionnaires }\end{array}$ & 2 sets $\times 8$ reps (12RM) & 2 sets $\times 10$ reps $(12 \mathrm{RM})$ \\
\hline \multicolumn{3}{|l|}{ Week 2 familiarisation and test } \\
\hline Day 4 & Day 5 & Day 6 \\
\hline
\end{tabular}

DXA, dual-energy X-ray absorptiometry; PLA, placebo; reps, repetitions; 1RM, one repetition maximum; SUPP, supplement; TUG, timed up and go.

has been previously proposed and currently being used in other exercise oncology trials. Moreover, any deviations to the protocol will be recorded in detail and reported in the final manuscript. Specific details of the programme are outlined in table 3 .

\section{Adherence}

Adherence to the resistance training protocol will be reported as a function of how much of the programme was actually completed versus what was initially

Table 3 Outline of the 12-week exercise programme characteristics

\begin{tabular}{llll}
\hline Weeks & Sets & Reps & Rest (seconds) \\
\hline $1-4$ & 3 & 12 & 60 \\
\hline $5-8$ & 3 & 10 & 60 \\
$9-10$ & 3 & 8 & 60 \\
$10-12$ & 4 & 8 & 60 \\
& Day 1 & Day 2 & Day 3 \\
Exercise & 1.Deadlift & 1.Push ups & 1.Deadlift \\
order & 2.Leg press & 2.Lat pulldown & 2.Leg press \\
& 3.Push ups* & 3.Chest press & 3.Push ups* \\
& 4.Seated row & 4.Seated row & 4.Seated row \\
& 5.Step up & 5.Shoulder & 5.Step up \\
& & press & \\
& 6.Chest press & 6.Deadlift & 6.Chest press \\
& 7.Pulldown & 7.Step up & 7.Pulldown \\
& 8.Shoulder & 8.Leg press & 8.Shoulder \\
& press & & press \\
\hline
\end{tabular}

*Push-ups will be performed on a Smith machine, where the initial height of the bar will be adjusted to a point where individuals can perform 12 repetitions. The exercise will be progressed by lowering the bar (15 cm intervals) when participants can successfully complete all repetitions. When participants can complete all repetitions on the floor with good technique, they will be progressed to a free weight bench press movement. planned. Specifically, volume load (sets x reps x weight) will be used to calculate planned training volume for each session. The training volume that was actually completed will be recorded. Any modifications to the exercise programme will be made at the discretion of the AEPs supervising the exercise programme. How and why sessions were modified will be recorded and reported in the final manuscript. The adherence to exercise will be reported as a ratio of completed to planned cumulative exercise, expressed as a percentage, previously outlined by Nilsen et $a l^{41}$ Additionally, reasons for missing a session will also be reported, with rescheduling of sessions permitted.

\section{SUPP and PLA protocol}

Participants in the SUPP group will receive $20 \mathrm{~g}$ /day of Cr monohydrate for 5 days, beginning on day 4 of the familiarisation/testing phase (immediately after randomisation, approximately 7 days prior to first training session), divided into four equal doses throughout the day. Participants will then be given single daily doses of $5 \mathrm{~g}$ for the duration of the 12-week training programme. This dosing protocol has been previously demonstrated to be safe and efficacious in older adults. ${ }^{18222527}$ Consequently, the loading phase prior to the beginning of the resistance training programme was included to ensure intramuscular Cr stores were saturated prior to resistance training to 'maximise' the likelihood of seeing an effect of supplementation. ${ }^{20}$ Participants in the PLA group will follow the same dosing protocol with dextrose. Dextrose is inert in terms of training adaptations and has been used as PLA in prior supplementation trials. ${ }^{18} 42$ Participants will be asked to dissolve the SUPPs in 200-300 $\mathrm{mL}$ of juice (orange or apple) to mask the solubility of $\mathrm{Cr}$ and taste of dextrose. To increase compliance, participants will be asked to return empty packets to the research team once a week. SUPP packages will be coded so that neither the investigators nor the participants will be aware of contents. 


\section{Statistical analysis}

To achieve $80 \%$ power at an alpha level of $\mathrm{p}<0.05$ (two tailed), 25 participants per group would be required to detect a mean difference in change between the two groups for FFM of $1.4 \mathrm{~kg}$ at the end of the 12-week intervention, based on our previous work in men receiving $\mathrm{ADT}^{11}$ and work by Brose $e t a l^{18}$ investigating changes in body composition and muscle strength with Cr supplementation and resistance training in older adults. To account for an attrition rate of $\sim 10 \%$ seen in prior trials, ${ }^{743}$ we aim to recruit 56 participants (SUPP n=28; PLA n=28). Statistical analysis will be conducted using SPSS (V.25, IBM). Normality of the distribution will be assessed using the Kolmogorov-Smirnov test. Summary descriptive statistics will be used for participant characteristics. Baseline characteristics will be assessed using independent t-tests or the Mann-Whitney U test, as appropriate. Separate group (Condition: SUPP, PL) x time (baseline, follow-up) repeated-measures analyses of variance or analyses of covariance, where appropriate, will be performed for each endpoint. Any data not normally distributed will be $\log$ transformed $(\mathrm{ln})$ for analysis. For categorical variables, Pearson $\mathrm{X}^{2}$ test will be used. Per-protocol analysis as well as an intention-to-treat approach with multiple imputations will be used to examine the data. Data will be deidentified and coded to classify which group the participant is in, without any ability to identify the individual. Identifiable documents will be destroyed, with deidentified information kept in a password protected electronic folder only accessible to the study team.

\section{Patient and public involvement}

EMRI regularly communicates with consumer representatives (individuals with cancer and their caregivers) with an acute understanding of cancer treatment-related side effects and the broader experience to provide input into the study design to ensure research supports the needs of this patient population and the community at large. Community representatives will remain active in their involvement of this study through regular updates with members of the study team and will be encouraged to offer suggestions on how to develop materials for plain language summaries and dissemination. Further, the clinicians on the study (CIT and RC) have high PCa patient caseloads that allow them to discuss the design of trials to target interventions to the needs of their patients.

\section{Ethics and dissemination}

Any protocol amendments will be submitted to the ethics committee for approval and these changes will be reflected in the trial registration. If the results of this trial demonstrate that $\mathrm{Cr}$ supplementation can augment adaptations of body composition, physical function and/ or psychosocial outcomes to resistance training, this study will provide effect sizes that will inform the design of subsequent definitive randomised controlled trials. The results of this study will be published in peer-reviewed journals and presented at various national and international conferences. The results of the trial will also be presented to all study participants (individually and collectively), and the EMRI team will work with local community members and support groups to develop materials for plain language summaries and dissemination.

\section{DISCUSSION}

Despite well-documented clinical benefits as a PCa treatment, ADT is consistently associated with profound worsening of muscle mass, neuromuscular strength and physical function in men with PCa. ${ }^{2-5}$ It has been postulated that reductions in muscle strength and physical function contribute to the cascade of physiological decline in men with $\mathrm{PCa}$ receiving ADT, ultimately resulting in a disability condition. ${ }^{64}{ }^{44}$ This contributes to a decline in activities of daily living, social activities and QOL. ${ }^{64}$ As a result, men with PCa are at a heightened risk of frailty, falls and fractures, and early mortality. ${ }^{356}$ Further, the decline in body composition is also associated with sarcopenic obesity, leading to increases in cardiometabolic risk factors. ${ }^{45}{ }^{46}$ Consequently, investigations into strategies that may improve musculoskeletal health is of critical importance in this population.

An ever-increasing body of literature supports the benefits of exercise, in particular resistance training, to counteract the loss of muscle mass and strength in men with PCa receiving ADT. ${ }^{9} 1047$ Recently, trials have emerged examining the potential for nutritional supplementation to augment adaptations to resistance training in men with PCa receiving ADT. ${ }^{48-50} \mathrm{Cr}$ is one of the most widely studied SUPPs, with increasing application across a breadth of populations. ${ }^{1751-58}$ Of particular relevance, $\mathrm{Cr}$ supplementation has been consistently demonstrated to enhance FFM in vulnerable populations that experience similar issues with loss of muscle mass to PCa, such as HIV, muscular dystrophy and older adults. ${ }^{23-30}$ Results from a recent meta-analysis by Chilibeck et $a \varphi^{\tilde{p}^{9}}$ demonstrate that Cr supplementation, in addition to resistance training, can result in $\sim 1.4 \mathrm{~kg}$ greater increase in FFM compared with resistance training alone in older adults. The authors also reported that $\mathrm{Cr}$ supplementation, in addition to resistance training, resulted in a greater increase in upper and lower body strength. ${ }^{59}$ Given the consistent reduction in FFM, muscle strength and physical function in men with PCa receiving ADT, particularly compared with apparently healthy controls, these results highlight the considerable therapeutic potential of Cr supplementation to more effectively ameliorate treatment-related side effects in this population. The safety of $\mathrm{Cr}$ is well established in a variety of apparently healthy and clinical populations, yet no research to date has investigated $\mathrm{Cr}$ supplementation in individuals with cancer. ${ }^{16}$ Consequently, the inclusion of questions on gastrointestinal distress and creatinine as a marker of kidney function will provide invaluable insight into the potential (or lack thereof) side effects of Cr supplementation in this population. 


\section{LIMITATIONS}

Several limitations of the study warrant discussion. The study is investigating Cr supplementation in individuals with PCa undergoing ADT, thus generalisability to other cancer populations is limited. ${ }^{18}$ Though a follow-up period to analyse the sustainability of outcomes would certainly be advantageous, limitations in funding and resources make it difficult to undertake in this study. As such the longer term benefits will not be elucidated.

\section{CONCLUSION}

This study will be the first specifically designed to investigate the efficacy of combining resistance training and $\mathrm{Cr}$ supplementation on FFM, muscle strength and physical function in men with PCa receiving ADT. Should the findings from the proposed study support the preliminary efficacy of Cr supplementation, appropriate effect sizes can be determined to help with the design of randomised controlled trials using Cr to target muscle loss in PCa.

\section{Author affiliations}

${ }^{1}$ Exercise Medicine Research Institute, Edith Cowan University, Joondalup, Western Australia, Australia

${ }^{2}$ School of Medical and Health Sciences, Edith Cowan University, Joondalup, Western Australia, Australia

${ }^{3}$ School of Human Movement and Nutrition Sciences, University of Queensland, Brisbane, Queensland, Australia

${ }^{4}$ Institute for Health Research, University of Notre Dame Australia, Fremantle, Western Australia, Australia

${ }^{5}$ Radiation Oncology, Genesis Care, Perth, Western Australia, Australia

${ }^{6}$ Department of Radiation Oncology, Sir Charles Gairdner Hospital, Perth, Western Australia, Australia

Acknowledgements The authors would like to thank the many contributors to Cancer Council Western Australia who have graciously funded this study. The authors would also like to thank our consumer representatives who have provided critical input into the design and development of this work.

Contributors All authors contributed to the design and development of the study protocol. CMF, KLK, RUN, NHH, DRT, RC, CIT and DAG collaboratively developed the concept and protocol, including analysis procedures and final reporting. CMF, KLK, RUN, NHH, DRT and DAG collectively designed the exercise protocol. KLK and CMF designed the supplementation elements of the protocol. CMF, KLK, RUN, NHH, DRT, $\mathrm{RC}, \mathrm{CIT}$ and DAG collectively determined the eligibility criteria for the trial. CMF and KLK designed the data collection documents and management aspects of the trial. CIT and RC will provide referrals to the study. CMF, KLK, RUN, NHH, DRT, RC, $\mathrm{CIT}$ and DAG contributed to writing, reviewing, editing and final approval of the study protocol per the International Committee for Medical Journal Editors (ICMJE) Recommendations.

Funding The authors have not declared a specific grant for this research from any funding agency in the public, commercial or not-for-profit sectors.

Competing interests None declared.

Patient consent for publication Not required.

Ethics approval Ethical approval was received from the Human Research Ethics Committee (HREC) at Edith Cowan University (ID: 22243 FAIRMAN).

Provenance and peer review Not commissioned; externally peer reviewed.

Open access This is an open access article distributed in accordance with the Creative Commons Attribution Non Commercial (CC BY-NC 4.0) license, which permits others to distribute, remix, adapt, build upon this work non-commercially, and license their derivative works on different terms, provided the original work is properly cited, appropriate credit is given, any changes made indicated, and the use is non-commercial. See: http://creativecommons.org/licenses/by-nc/4.0/.

\section{REFERENCES}

1. Rhee H, Gunter JH, Heathcote P, et al. Adverse effects of androgendeprivation therapy in prostate cancer and their management. BJU Int 2015;115(Suppl.):3-13.

2. Boxer RS, Kenny AM, Dowsett R, et al. The effect of 6 months of androgen deprivation therapy on muscle and fat mass in older men with localized prostate cancer. Aging Male 2005;8:207-12.

3. Galvão DA, Spry NA, Taaffe DR, et al. Changes in muscle, fat and bone mass after 36 weeks of maximal androgen blockade for prostate cancer. BJU Int 2008;102:44-7.

4. Gonzalez BD, Jim HSL, Small BJ, et al. Changes in physical functioning and muscle strength in men receiving androgen deprivation therapy for prostate cancer: a controlled comparison. Support Care Cancer 2016;24:2201-7.

5. Levy ME, Perera S, van Londen GJ, et al. Physical function changes in prostate cancer patients on androgen deprivation therapy: a 2-year prospective study. Urology 2008;71:735-9.

6. Galvão DA, Taaffe DR, Spry N, et al. Exercise can prevent and even reverse adverse effects of androgen suppression treatment in men with prostate cancer. Prostate Cancer Prostatic Dis 2007;10:340-6.

7. Galvão DA, Spry N, Denham J, et al. A multicentre year-long randomised controlled trial of exercise training targeting physical functioning in men with prostate cancer previously treated with androgen suppression and radiation from TROG 03.04 radar. Eur Urol 2014;65:856-64.

8. Galvão DA, Taaffe DR, Spry N, et al. Combined resistance and aerobic exercise program reverses muscle loss in men undergoing androgen suppression therapy for prostate cancer without bone metastases: a randomized controlled trial. JCO 2010;28:340-7.

9. Padilha CS, Marinello PC, Galvão DA, et al. Evaluation of resistance training to improve muscular strength and body composition in cancer patients undergoing neoadjuvant and adjuvant therapy: a meta-analysis. J Cancer Surviv 2017;11:339-49.

10. Focht BC, Lucas AR, Grainger E, et al. Effects of a group-mediated exercise and dietary intervention in the treatment of prostate cancer patients undergoing androgen deprivation therapy: results from the IDEA-P trial. Ann Behav Med 2018;52:412-28.

11. Newton RU, Galvão DA, Spry N, et al. Exercise mode specificity for preserving spine and hip bone mineral density in prostate cancer patients. Med Sci Sports Exerc 2019;51:607-14.

12. Fried LP, Ferrucci L, Darer J, et al. Untangling the concepts of disability, frailty, and comorbidity: implications for improved targeting and care. J Gerontol A Biol Sci Med Sci 2004;59:M255-M263.

13. Gualano B, Artioli GG, Poortmans JR, et al. Exploring the therapeutic role of creatine supplementation. Amino Acids 2010;38:31-44.

14. Shachar SS, Deal AM, Weinberg M, et al. Body composition as a predictor of toxicity in patients receiving anthracycline and taxanebased chemotherapy for early-stage breast cancer. Clin Cancer Res 2017;23:3537-43.

15. Shachar SS, Williams GR, Muss HB, et al. Prognostic value of sarcopenia in adults with solid tumours: a meta-analysis and systematic review. Eur J Cancer 2016;57:58-67.

16. Fairman $\mathrm{CM}$, Kendall $\mathrm{KL}$, Hart $\mathrm{NH}$, et al. The potential therapeutic effects of creatine supplementation on body composition and muscle function in cancer. Crit Rev Oncol Hematol 2019;133:46-57.

17. Aguiar AF, Januário RSB, Junior RP, et al. Long-Term creatine supplementation improves muscular performance during resistance training in older women. Eur J Appl Physiol 2013;113:987-96.

18. Brose A, Parise G, Tarnopolsky MA. Creatine supplementation enhances isometric strength and body composition improvements following strength exercise training in older adults. J Gerontol A Biol Sci Med Sci 2003;58:B11-B19.

19. Buford TW, Kreider RB, Stout JR, et al. International Society of sports nutrition position stand: creatine supplementation and exercise. J Int Soc Sports Nutr 2007;4:6.

20. Kreider RB, Kalman DS, Antonio J, et al. International Society of sports nutrition position stand: safety and efficacy of creatine supplementation in exercise, sport, and medicine. J Int Soc Sports Nutr 2017;14:18.

21. Lanhers C, Pereira B, Naughton G, et al. Creatine supplementation and lower limb strength performance: a systematic review and metaanalyses. Sports Med 2015;45:1285-94.

22. Devries MC, Phillips SM. Creatine supplementation during resistance training in older adults-a meta-analysis. Med Sci Sports Exerc 2014;46:1194-203.

23. Gotshalk LA, Kraemer WJ, Mendonca MAG, et al. Creatine supplementation improves muscular performance in older women. Eur J Appl Physiol 2008;102:223-31.

24. Gotshalk LA, Volek JS, Staron RS, et al. Creatine supplementation improves muscular performance in older men. Med Sci Sports Exerc 2002;34:537-43. 
25. Gualano B, Macedo AR, Alves CRR, et al. Creatine supplementation and resistance training in vulnerable older women: a randomized double-blind placebo-controlled clinical trial. Exp Gerontol 2014:53:7-15.

26. Lobo DM, Tritto AC, da Silva LR, et al. Effects of long-term low-dose dietary creatine supplementation in older women. Exp Gerontol 2015;70:97-104.

27. Pinto CL, Botelho PB, Carneiro JA, et al. Impact of creatine supplementation in combination with resistance training on lean mass in the elderly. J Cachexia Sarcopenia Muscle 2016;7:413-21.

28. Sakkas GK, Mulligan K, Dasilva M, et al. Creatine fails to augment the benefits from resistance training in patients with HIV infection: a randomized, double-blind, placebo-controlled study. PLoS One 2009;4:e4605.

29. Stout JR, Sue Graves B, Cramer JT, et al. Effects of creatine supplementation on the onset of neuromuscular fatigue threshold and muscle strength in elderly men and women (64 - 86 years). $J$ Nutr Health Aging 2007;11:459-64.

30. Tarnopolsky MA, Mahoney DJ, Vajsar J, et al. Creatine monohydrate enhances strength and body composition in Duchenne muscular dystrophy. Neurology 2004;62:1771-7.

31. de Campos-Ferraz PL, Andrade I, das Neves W, et al. An overview of amines as nutritional supplements to counteract cancer cachexia. $J$ Cachexia Sarcopenia Muscle 2014;5:105-10.

32. Harris RC, Söderlund K, Hultman E. Elevation of creatine in resting and exercised muscle of normal subjects by creatine supplementation. Clin Sci 1992;83:367-74.

33. Olsen S, Aagaard P, Kadi F, et al. Creatine supplementation augments the increase in satellite cell and myonuclei number in human skeletal muscle induced by strength training. $J$ Physiol 2006;573:525-34.

34. Hart NH, Nimphius S, Spiteri T, et al. Segmental musculoskeletal examinations using dual-energy X-ray absorptiometry (DXA): positioning and analysis considerations. J Sports Sci Med 2015;14:620-6.

35. Jeldres C, Cullen J, Hurwitz LM, et al. Prospective quality-of-life outcomes for low-risk prostate cancer: active surveillance versus radical prostatectomy. Cancer 2015;121:2465-73.

36. Nolte S, Liegl G, Petersen MA, et al. General population normative data for the EORTC QLQ-C30 health-related quality of life questionnaire based on 15,386 persons across 13 European countries, Canada and the Unites states. Eur J Cancer 2019;107:153-63.

37. van Andel G, Bottomley A, Fosså SD, et al. An international field study of the EORTC QLQ-PR25: a questionnaire for assessing the health-related quality of life of patients with prostate cancer. Eur $J$ Cancer 2008;44:2418-24.

38. Butt Z, Lai J-S, Rao D, et al. Measurement of fatigue in cancer, stroke, and HIV using the functional assessment of chronic illness therapy - fatigue (FACIT-F) scale. J Psychosom Res 2013;74:64-8.

39. Kraemer WJ, Ratamess NA, French DN. Resistance training for health and performance. Curr Sports Med Rep 2002;1:165-71.

40. American College of Sports Medicine. American College of sports medicine position stand. progression models in resistance training for healthy adults. Med Sci Sports Exerc 2009;41:687-708.

41. Nilsen TS, Scott JM, Michalski M, et al. Novel methods for reporting of exercise dose and adherence: an exploratory analysis. Med Sci Sports Exerc 2018;50:1134-41.

42. Willoughby DS, Stout JR, Wilborn CD. Effects of resistance training and protein plus amino acid supplementation on muscle anabolism, mass, and strength. Amino Acids 2007;32:467-77.
43. Newton RU, Jeffery E, Galvão DA, et al. Body composition, fatigue and exercise in patients with prostate cancer undergoing androgendeprivation therapy. BJU Int 2018;122:986-93.

44. Winters-Stone KM, Dobek JC, Bennett JA, et al. Resistance training reduces disability in prostate cancer survivors on androgen deprivation therapy: evidence from a randomized controlled trial. Arch Phys Med Rehabil 2015:96:7-14.

45. Aversa A, Francomano D, Lenzi A. Cardiometabolic complications after androgen deprivation therapy in a man with prostate Cancer: effects of 3 years intermittent testosterone supplementation. Front Endocrinol 2012;3:17.

46. Turner L, Poole K, Faithfull S, et al. Current and future strategies for the nutritional management of cardiometabolic complications of androgen deprivation therapy for prostate cancer. Nutr Res Rev 2017;30:220-32.

47. Galvão DA, Taaffe DR, Spry N, et al. Exercise preserves physical function in prostate cancer patients with bone metastases. Med Sci Sports Exerc 2018;50:393-9.

48. Dawson JK, Dorff TB, Todd Schroeder E, et al. Impact of resistance training on body composition and metabolic syndrome variables during androgen deprivation therapy for prostate cancer: a pilot randomized controlled trial. BMC Cancer 2018;18:368.

49. Kiwata JL, Dorff TB, Todd Schroeder E, et al. A pilot randomised controlled trial of a periodised resistance training and protein supplementation intervention in prostate cancer survivors on androgen deprivation therapy. BMJ Open 2017;7:e016910.

50. Owen PJ, Daly RM, Livingston PM, et al. Efficacy of a multicomponent exercise programme and nutritional supplementation on musculoskeletal health in men treated with androgen deprivation therapy for prostate cancer (impact): study protocol of a randomised controlled trial. Trials 2017:18:451.

51. Al-Ghimlas F, Todd DC. Creatine supplementation for patients with COPD receiving pulmonary rehabilitation: a systematic review and meta-analysis. Respirology 2010;15:785-95.

52. Andrews R, Greenhaff $P$, Curtis $S$, et al. The effect of dietary creatine supplementation on skeletal muscle metabolism in congestive heart failure. Eur Heart J 1998;19:617-22.

53. Bemben MG, Witten MS, Carter JM, et al. The effects of supplementation with creatine and protein on muscle strength following a traditional resistance training program in middle-aged and older men. J Nutr Health Aging 2010;14:155-9.

54. Bender A, Koch W, Elstner M, et al. Creatine supplementation in Parkinson disease: a placebo-controlled randomized pilot trial. Neurology 2006;67:1262-4.

55. Candow DG, Little JP, Chilibeck PD, et al. Low-Dose creatine combined with protein during resistance training in older men. Med Sci Sports Exerc 2008;40:1645-52.

56. Cañete $S$, San Juan AF, Pérez M, et al. Does creatine supplementation improve functional capacity in elderly women? $\mathrm{J}$ Strength Cond Res 2006;20:22-8.

57. Cornelissen VA, Defoor JGM, Stevens A, et al. Effect of creatine supplementation as a potential adjuvant therapy to exercise training in cardiac patients: a randomized controlled trial. Clin Rehabil 2010;24:988-99

58. Gualano B, de Salles Painelli V, Roschel H, et al. Creatine supplementation does not impair kidney function in type 2 diabetic patients: a randomized, double-blind, placebo-controlled, clinical trial. Eur J Appl Physiol 2011;111:749-56.

59. Chilibeck PD, Kaviani M, Candow DG, et al. Effect of creatine supplementation during resistance training on lean tissue mass and muscular strength in older adults: a meta-analysis. Open Access $J$ Sports Med 2017;8:213-26. 